\title{
Simultaneous Pancreatitis and Myocarditis in Childhood-Onset Systemic Lupus Erythematous
}

\author{
Guang Mei JIANG (D), Peng HU(D), Yue WU(1)
}

Departent of Pediatrics, The First Affiliated Hospital of Anhui Medical University, Hefei, China

An 11-year-old girl was admitted to our department with a six-month history of anorexia and rashes on her face and extremities (Figure 1). On admission, her axillary temperature was $37.7^{\circ} \mathrm{C}$, heart rate was 130 beats/minute, blood pressure was $82 / 47 \mathrm{mmHg}$, respiratory rate was 22 breaths/minute, and oxygen saturation was 95\%. Cardiac examination revealed a soft systolic murmur at the apex with low heart sounds. She had malar rash, discoid rash, hepatosplenomegaly, and edema of the lower extremities. The subsequent autoimmune and serologic workup came back positive for anti-nuclear antibody (ANA) at a titer of 1:3200, positive anti-double stranded deoxyribonucleic acid, anti-Smith antibody, and low complements $(0.28 \mathrm{~g} / \mathrm{L})$ and
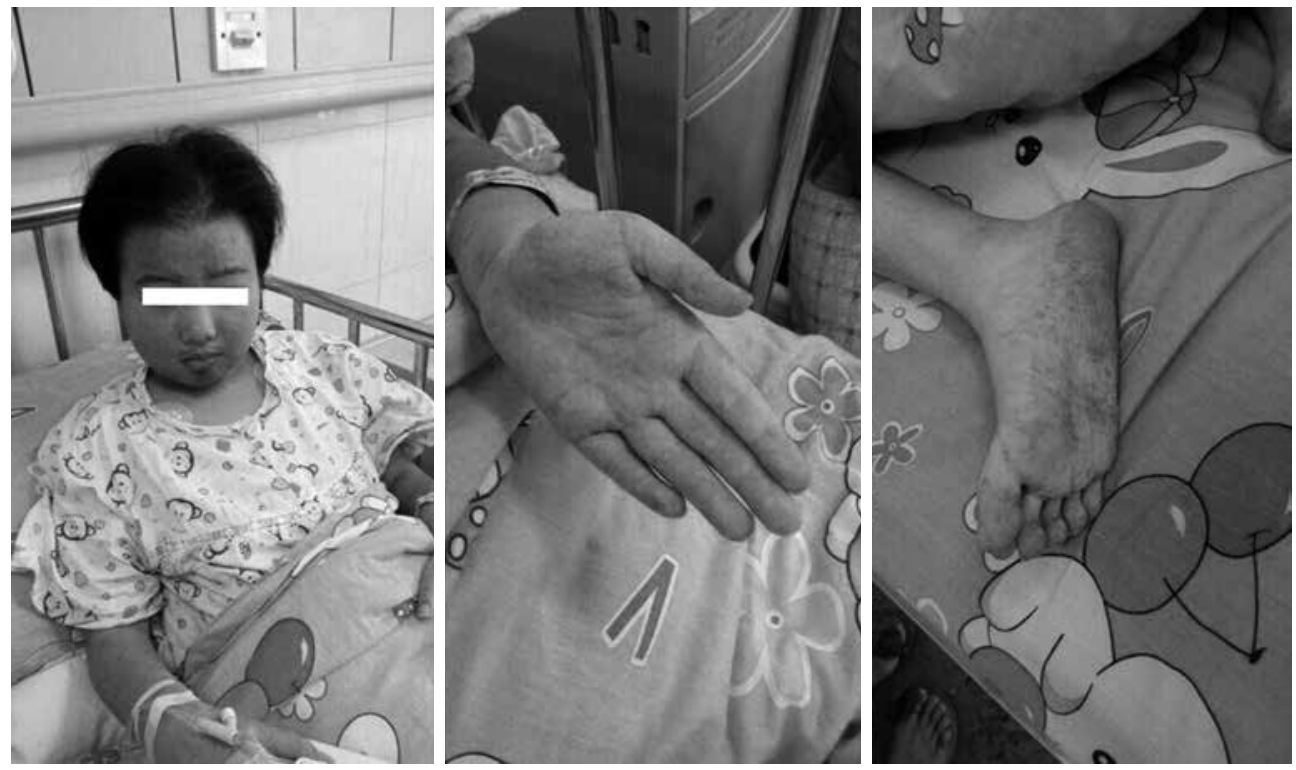

Figure 1. Widespread rashes on face and extremities.

Received: July 24, 2017 Accepted: December 13, 2017 Published online: January 18, 2017

Correspondence: Peng Hu, MD. Departent of Pediatrics, The First Affiliated Hospital of Anhui Medical University, 230022 Hefei, China. Tel: +86055162922058 e-mail: hupeng28@aliyun.com 


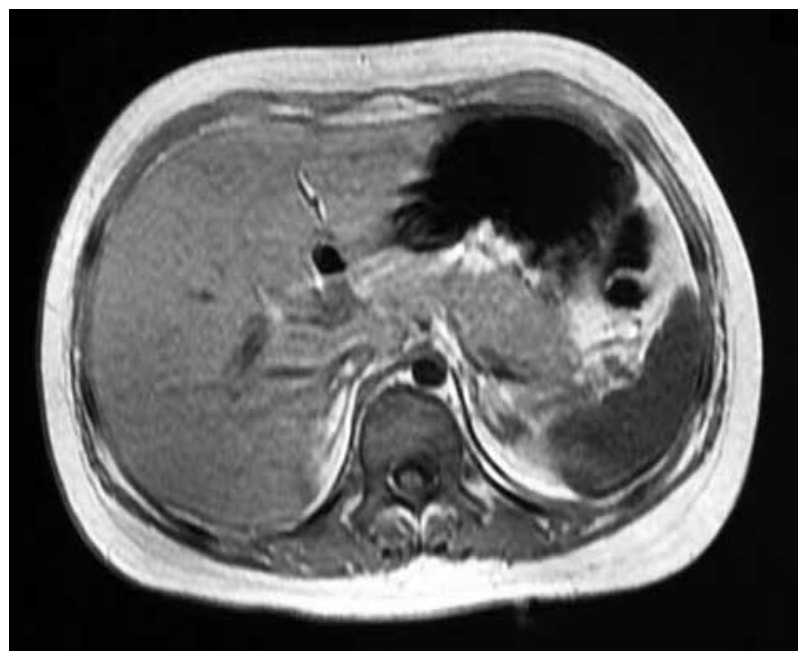

Figure 2. Abdominal computed tomography scan demonstrated swollen pancreas but no gallbladder stones.

complement 4 (C4) (0.03 g/L). Other laboratory studies exhibited anemia (red blood cell $3.1 \times 10^{12} / \mathrm{L}$, hemoglobin $99 \mathrm{~g} / \mathrm{L}$ ), proteinuria (24-hour urine protein $300 \mathrm{mg} /$ day), elevation of acute-phase reactants (C-reactive protein [CRP] $132 \mathrm{mg} / \mathrm{L}$, erythrocyte sedimentation rate $54 \mathrm{~mm}$ /hour), hyperamylasemia (208 units/L), hyperlipasemia (1556 units/L), and raised creatine kinase (CK, 375 units/L) and CK-MB (83 units/L). Electrocardiogram revealed sinus tachycardia with $\mathrm{T}$ wave inversion. Echocardiography showed normal left ventricular size but global hypokinesia with an ejection fraction (EF) of 37\%. Abdominal computed tomography (CT) demonstrated swollen pancreas but no gallbladder stones (Figure 2). Renal biopsy revealed diffuse proliferative glomerulonephritis Class IV-G. Our patient was diagnosed as having systemic lupus erythematous (SLE) complicated by pancreatitis and myocarditis. Systemic lupus erythematous disease activity index (SLEDAI) score was 24. She was treated with a course of pulse methylprednisolone $10 \mathrm{mg} / \mathrm{kg} / \mathrm{day}$ for three days and subsequently converted to oral prednisone $(60 \mathrm{mg} /$ day $)$. In addition, several symptomatic treatments such as bowel rest, nutritional support, somatostatin, and fructose1,6-diphosphate were also adopted. One month later, intravenous cyclophosphamide $500 \mathrm{mg} / \mathrm{m}^{2}$ was given. Two months later, repeated echocardiography showed a marked improvement in EF, abdominal CT revealed a less swollen pancreas, and SLEDAI score was 2. The patient was discharged with normal pancreatic (amylase 109 units/L, lipase 285 units/L) and myocardial enzymes (CK 29 units/L, CK-MB 11 units/L).

Systemic lupus erythematous is a chronic, autoimmune disease characterized by the presence of a plethora of autoantibodies, immune complex formation, and multiple organ involvement. The American Rheumatism Association criteria for SLE are still the most commonly used criteria for diagnosis. ${ }^{1}$ In this report, our patient fulfilled six criteria (malar rash, discoid rash, renal disorder, hematologic disorder, immunologic disorder, and ANA), thus the diagnosis of SLE could be established undoubtedly. The major reason triggering us to present this case was the co-existed myocarditis and pancreatitis secondary to SLE. Secondly, CRP was skyrocketing in parallel with multiple organ dysfunction. An Indian retrospective study enrolling 141 SLE cases suggested that a positive correlation was observed between CRP and SLEDAI. ${ }^{2}$ Therefore, CRP can reflect the disease activity of SLE to some extent.

Myocarditis is an uncommon feature of SLE, and its clinical manifestations are variable, ranging from asymptomatic presentation to cardiogenic shock. In the present report, our patient was diagnosed with myocarditis on the basis of hypotension, cardiac auscultation, diffuse T-wave inversion on electrocardiogram, elevated cardiac markers, and echocardiographic findings. Particularly, the presence of global hypokinesia with a low EF on echocardiography is strong evidence towards the diagnosis. ${ }^{3}$ However, myocardial biopsy is used infrequently because of perceived risks and the low specificity of the histology. Besides myocarditis, SLE in this case was also complicated by pancreatitis, according to elevation of pancreatic enzymes and characteristic imaging findings. Although the exact mechanism has not been revealed yet, the pathogenesis of SLE-associated pancreatitis may include vasculitis, hemolysis, micro-thrombus formation, anti-pancreatic antibodies, and inflammation due to T-cell infiltration and complement activation. ${ }^{4}$

Despite diverse potential contributors to myocarditis and pancreatitis, recent studies support that SLE itself serves as the most important etiologic factor for these two 
complications. ${ }^{5}$ In addition, corticosteroid therapy is rather controversial. However, accumulating evidence suggests that the immunosuppressive effect of corticosteroid is essential not only to improve the multiple organ involvements, but to decrease the mortality due to SLE. ${ }^{6}$ On this background, our patient received a course of high-dose systemic corticosteroid for three days with subsequent dose tapering and obtained a satisfactory prognosis.

\section{Acknowledgments}

The authors would like to gratefully acknowledge the most helpful comments on this paper received from Professor Bo Hu, Department of Pediatrics, The first Affiliated Hospital of Anhui Medical University, Hefei, People's Republic of China.

\section{Declaration of conflicting interests}

The authors declared no conflicts of interest with respect to the authorship and/or publication of this article.

\section{Funding}

The authors received no financial support for the research and/or authorship of this article.

\section{REFERENCES}

1. Tan EM, Cohen AS, Fries JF, Masi AT, McShane DJ, Rothfield NF, et al. The 1982 revised criteria for the classification of systemic lupus erythematosus. Arthritis Rheum 1982;25:1271-7.

2. Umare V, Nadkarni A, Nadkar M, Rajadhyksha A, Khadilkar P, Ghosh K, et al. Do high sensitivity C-reactive protein and serum interleukin-6 levels correlate with disease activity in systemic lupus erythematosuspatients? J Postgrad Med 2017;63:92-5.

3. Gupta A, Singh S, Minz RW, Radotra BD, Ahluwalia $\mathrm{J}$, Grover A. Lupus myocarditis in children. Ann Rheum Dis 2004;63:745-6.

4. Masoodi I. The simultaneous incidence of acute pancreatitis and autoimmune hemolytic anemia: a rare duo in a patient with SLE. Ger Med Sci 2014;12:Doc12.

5. Richer O, Ulinski $\mathrm{T}$, Lemelle I, Ranchin $\mathrm{B}$, Loirat $\mathrm{C}$, Piette $\mathrm{JC}$, et al. Abdominal manifestations in childhood-onset systemic lupus erythematosus. Ann Rheum Dis. 2007;66:174-8.

6. Tian XP, Zhang X. Gastrointestinal involvement in systemic lupus erythematosus: insight into pathogenesis, diagnosis and treatment. World J Gastroenterol 2010;16:2971-7. 